\title{
The relationship between obesity and diabetic nephropathy in China
}

\author{
Hui-Mei Chen*, Wen-Wen Shen, Yong-Chun Ge, Yi-De Zhang, Hong-Lang Xie and Zhi-Hong Liu*
}

\begin{abstract}
Background: The epidemic of diabetic nephropathy (DN) has been paralleled by rapid increases in both obesity and diabetes in China. The aim of this study was to investigate the natural history of DN and the association of obesity and renal function with diabetes.

Methods: In total, 264 patients with renal biopsy-confirmed DN were examined from 2002 to 2008 and followed up to June 2008 in our institute. Among these, 129 patients were classified into a Kidney Disease Outcomes Quality Initiative (K/DOQI) stage I subgroup. Weight status, clinico-histopathological features, the development of end-stage renal disease (ESRD) and increased proteinuria were evaluated at the baseline of biopsy and during the follow up. Lean, overweight and obese phenotypes were defined as body mass index (BMI) less than $25 \mathrm{~kg} / \mathrm{m}^{2}, 25-28 \mathrm{~kg} / \mathrm{m}^{2}$, and more than $28 \mathrm{~kg} / \mathrm{m}^{2}$ over, respectively.

Results: In the patients with renal biopsy-confirmed DN, BMI was $25.5 \pm 3.39 \mathrm{~kg} / \mathrm{m}^{2}$, with $122(46.2 \%), 83$ (31.4\%) and 59 (22.3\%) having lean, overweight and obese phenotypes, respectively. Mean proteinuria was $3.09 \pm 2.32 \mathrm{~g} / 24 \mathrm{~h}$, serum creatinine was $2.02 \pm 2.02 \mathrm{mg} / \mathrm{dL}$, and creatinine clearance rate $(C \mathrm{cr})$ was $96.0 \pm 54.0 \mathrm{~mL} / \mathrm{min} / 1.73 \mathrm{~m}^{2}$. Compared with obese patients, lean patients had a lower $\mathrm{Ccr}$, a higher percentage of anemia, more renal lesions and higher risk for ESRD ( $H R=1.812, P=0.048)$. The weight in obese patients decreased significantly after 27 months, and lean patients had a longer duration of diabetes than obese patients. Regarding patients at K/DOQI stage I, patients with DN showed similar duration of diabetes regardless of weight status. Minimal weight loss was recorded in obese patients during follow-up, and they exhibited greater glomerular hyperfiltration and higher risk for increased proteinuria $(H R=2.872$, $P=0.014)$ than lean patients.
\end{abstract}

Conclusions: In China, obesity is common in DN patients undergoing biopsy. Initial high levels of proteinuria and subsequent weight loss are the major characteristics of the natural course of DN. Obesity contributed to increased proteinuria at an early stage, while the lean phenotype was associated with ESRD development, especially at the later stages.

Keywords: Diabetic nephropathy, ESRD, Obesity, Proteinuria

\section{Background}

Obesity has become a serious worldwide problem. More than 300 million adults are classified as obese and the global number is predicted to reach 700 million by 2015 . In the past, obesity was not a common condition among the population of China; however, this is no longer the case [1]. Obesity and diabetes have become a growing challenge, and one million new diabetic cases are reported in China every year. Increasing evidence suggests that obesity

\footnotetext{
* Correspondence: chenhuimei@nju.edu.cn; zhihong-liu@hotmail.com Research Institute of Nephrology, Jinling Hospital, Nanjing University School of Medicine, Nanjing 210002, China
}

\section{Biomed Central}

is a risk factor for diabetes and chronic kidney diseases. As a marker of obesity, high body mass index (BMI) has been reported to be related with diabetic nephropathy (DN) and end-stage renal disease (ESRD) [2]. A high prevalence of DN and ESRD has recently been demonstrated among Asians. During the past two decades, the prevalence of DN has dramatically increased in China $[3,4]$, although the obesity profile among DN patients and the natural history of DN are not available in this region.

Obesity has been shown to aggravate renal injury associated with diabetes, although this effect is not consistent in previous studies. The evidence indicating a 
relationship between obesity and ESRD is paradoxical $[5,6]$. Tokashiki et al. showed that decreased BMI was the independent risk factor for chronic kidney disease, and the effect of obesity on renal survival varied with ethnicity $[1,7]$. Without intensive analysis, the possible role of obesity and weight loss on kidney function is still uncertain among Chinese patients with DN.

The first aim of our study was to explore the natural history of DN in Chinese patients. The second aim was to examine the impact of obesity on renal function in such a population. We screened patients presenting with type 2 diabetes, proteinuria, and biopsy-confirmed DN in our institute, and demonstrated an association of the obesity, weight loss with $\mathrm{DN}$, and BMI with renal injury at different stages of diabetes. Obesity and overweight were defined according to the criteria outlined by the Working Group on Obesity of China [8]. The renal stages were evaluated by K/DOQI (Kidney Disease Outcomes Quality Initiative) guidelines.

\section{Methods}

\section{Study population}

Information on 15,141 cases was retrieved from patients, who underwent renal biopsy at a single unit during the period from July 2002 to June 2008 (Research Institute of Nephrology, Nanjing University School of Medicine, P.R. China). In this institute, the indications for renal biopsy included proteinuria and/or hematuria and/or renal insufficiency, with or without coexisting systemic disease [9]. The biopsies were performed by a group of clinicians, and renal biopsy specimens were examined by experienced nephrologists using techniques including light microscopy, immunohistology, and electron microscopy. Final diagnosis was made on the basis of clinical and histological features.

Written informed consent was obtained from all subjects prior to participation in the experimental protocol. The study was approved by the institutional review board of Nanjing University School of Medicine, and consistent with the Declaration of Helsinki. Initially, 757 patients with renal biopsy-confirmed DN were included, according to the following criteria: 1) type 2 diabetes mellitus (Standards of Medical Care in Diabetes, 2008, ADA [10]); 2) urinary protein excretion $>400 \mathrm{mg} / 24 \mathrm{~h}$; and 3) typical DN lesions in the glomeruli, tubulointerstitium or blood vessels [11]. Patients with concomitant conditions such as IgA nephropathy and secondary renal damage due to hypertension were excluded $[12,13]$. All patients were recommended to follow-up in the out-patient center every 3 months; however, information was obtained for only 264 subjects to June 2008. The base characteristics of these 264 subjects were similar to those of the 757 subjects that were initially included (Additional file 1: Table S1).

\section{Definitions}

BMI was calculated as weight $(\mathrm{kg})$ divided by height ${ }^{2}\left(\mathrm{~m}^{2}\right)$. Based on the cut-off points in Chinese adults [8], BMI was classified as follows: obese, $\geq 28.0 \mathrm{~kg} / \mathrm{m}^{2}$; overweight, 25.0 $27.9 \mathrm{~kg} / \mathrm{m}^{2}$; lean $<25.0 \mathrm{~kg} / \mathrm{m}^{2}$. The proteinuria per 24 hours and creatinine clearance rate $(\mathrm{Ccr})$ were evaluated by 24-hour urine collection at the time of biopsy, and Ccr were calculated with the standard formula: urine creatinine $(\mathrm{mg} / \mathrm{dL}) \times$ urine volume $(\mathrm{mL} / \mathrm{min}) /$ serum creatinine $(\mathrm{mg} / \mathrm{dL})$ and adjusted for body surface [14]. Other definitions were used: 1) nephrotic range proteinuria, 24-hour urine protein excretion $\geq 3.5 \mathrm{~g} ; 2$ ) hypoalbuminemia, serum albumin levels $\leq 3.5 \mathrm{~g} / \mathrm{L} ; 3) \mathrm{K} / \mathrm{DOQ} 1$ stage I, Ccr $>90 \mathrm{~mL} / \mathrm{min} / 1.73 \mathrm{~m}^{2}$; and 3) renal insufficiency, Ccr $<60 \mathrm{~mL} / \mathrm{min} / 1.73 \mathrm{~m}^{2}$. Insulin resistance was assessed using the Homeostasis Model Assessment of Insulin Resistance formula: fasting serum insulin $(\mu \mathrm{U} / \mathrm{mL}) \times$ fasting plasma glucose $(\mathrm{mmol} / \mathrm{L}) / 22.5$ [15]. Anemia was defined as hemoglobin $<12$ and $<11 \mathrm{~g} / \mathrm{dL}$ in men and women, respectively.

Periodic acid-Schiff-stained paraffin-embedded sections at $\times 400$ magnification were used to estimate pathologic lesions $[16,17]$. The percentages of globally sclerotic glomeruli and Kimmelstiel-Wilson (K-W) nodules were recorded from 50 glomerular sections for each patient. A semiquantitative assessment of renal damage was performed according to mesangial proliferation, interstitial fibrosis, tubular atrophy and arteriolar hyalinosis [18].

The progression of renal injury was defined by the development of ESRD and increased proteinuria. The primary endpoint, ESRD, was defined as the requirement for permanent renal replacement therapy or serum creatinine exceeding $6.0 \mathrm{mg} / \mathrm{dL}$ for more than 1 month without other causes of renal dysfunction. The secondary endpoint, increased proteinuria, was defined as a $50 \%$ increase from the baseline level of proteinuria.

\section{Statistical analysis}

Statistical analysis was performed using SPSS, version 11.0 (SPSS Inc, Chicago, IL). Quantitative variables were expressed as mean $\pm \mathrm{SD}$ and compared by one-way ANOVA, followed with Least-Significant Difference (LSD) tests between each pair of groups. In the absence of a normal distribution, log-transformed data values were used for analysis and the Mann-Whitney test $U$ test was used when necessary. Qualitative variables were described as sample size (number of cases) and percentage (\%), which were analyzed by chi-square test or Fisher's exact test. Cumulative incidence of ESRD or disease progression was calculated using Kaplan-Meier survival probabilities (1- survival probabilities). Adjusted relative risks of mortality were calculated using Cox regression analysis. Two-tailed $P$-values less than 0.05 were considered statistically significant. 
Table 1 Epidemiological characteristics of subjects with diabetic nephropathy

\begin{tabular}{|c|c|c|c|c|c|}
\hline & Total & $\begin{array}{c}\text { Lean Group } \\
\mathrm{BMI}<25\end{array}$ & $\begin{array}{c}\text { Overweight Group } \\
25 \leq \mathrm{BMI}<28\end{array}$ & $\begin{array}{c}\text { Obese Group } \\
\mathrm{BMI} \geq \mathbf{2 8}\end{array}$ & $P$ value \\
\hline No. of patients & 264 & 122 & 83 & 59 & \\
\hline $\mathrm{BMI}\left(\mathrm{kg} / \mathrm{m}^{2}\right)$ & $25.5 \pm 3.39$ & $22.6 \pm 1.59$ & $26.4 \pm 0.85$ & $31.3 \pm 1.91$ & - \\
\hline Age (years) & $53.1 \pm 9.06$ & $54.1 \pm 9.34$ & $54.0 \pm 8.97$ & $53.1 \pm 8.62$ & 0.787 \\
\hline Male sex (\%) & 154(58.3\%) & $73(59.8 \%)$ & $47(56.6 \%)$ & $34(57.6 \%)$ & 0.894 \\
\hline Known duration of diabetes (months) & $111 \pm 73.7$ & $123 \pm 74.8$ & $110 \pm 74.9$ & $84.0 \pm 62.1^{* * \neq}$ & 0.002 \\
\hline Known duration of proteinuria (months) & $27.7 \pm 41.7$ & $23.7 \pm 29.7$ & $28.0 \pm 39.7$ & $27.8 \pm 56.8$ & 0.698 \\
\hline Fasting glucose (mg/dL) & $124 \pm 44$ & $125 \pm 48$ & $117 \pm 34$ & $128 \pm 51$ & 0.338 \\
\hline $\mathrm{HbA1c}(\%)$ & $6.66 \pm 1.33$ & $6.81 \pm 1.50$ & $6.48 \pm 1.20$ & $6.61 \pm 1.11$ & 0.230 \\
\hline Mean blood pressure (mmHg) & $108 \pm 14.2$ & $109 \pm 14.5$ & $105 \pm 13.3$ & $109 \pm 14.8$ & 0.139 \\
\hline Proteinuria (g/24 h) & $3.09 \pm 2.32$ & $3.19 \pm 2.19$ & $2.97 \pm 2.46$ & $3.08 \pm 2.69$ & 0.821 \\
\hline$\geq 3.5 \mathrm{~g} / 24 \mathrm{~h}(\%)$ & $103(37.4 \%)$ & $48(39.3 \%)$ & $32(38.6 \%)$ & $23(39.0 \%)$ & 0.927 \\
\hline Serum albumin (g/dL) & $3.45 \pm 0.74$ & $3.36 \pm 0.69$ & $3.46 \pm 0.80$ & $3.66 \pm 0.74^{*}$ & 0.017 \\
\hline$<3.5 \mathrm{~g} / \mathrm{dL}(\%)$ & $141(53.5 \%)$ & 78 (63.9\%) & $37(44.6 \%)^{* *}$ & $26(44.1 \%)^{* *}$ & 0.007 \\
\hline Serum creatinine $(\mathrm{mg} / \mathrm{dL})$ & $2.02 \pm 2.02$ & $2.39 \pm 2.27$ & $1.52 \pm 1.14^{* *}$ & $2.00 \pm 2.36$ & 0.010 \\
\hline $\mathrm{Ccr}\left(\mathrm{mL} / \mathrm{min} / 1.73 \mathrm{~m}^{2}\right)$ & $97.8 \pm 53.4$ & $81.6 \pm 51.0$ & $114 \pm 57.6^{* *}$ & $109 \pm 64.8^{* *}$ & $<0.001$ \\
\hline$<60 \mathrm{~mL} / \mathrm{min} / 1.73 \mathrm{~m}^{2}(\%)$ & $85(32.2 \%)$ & $52(42.6 \%)$ & $18(21.7 \%)^{* *}$ & $15(25.4 \%)^{* *}$ & $<0.001$ \\
\hline Uric acid $(\mu \mathrm{mol} / \mathrm{L})$ & $400 \pm 108$ & $391 \pm 110$ & $401 \pm 101$ & $418 \pm 113$ & 0.340 \\
\hline Hemoglobin (g/dL) & $11.2 \pm 2.6$ & $10.4 \pm 2.3$ & $11.7 \pm 2.5^{* *}$ & $12.3 \pm 2.7^{* *}$ & $<0.001$ \\
\hline Anemia (\%) & $140(54.9 \%)$ & $84(68.9 \%)$ & $39(47.0 \%) * *$ & $17(34.7 \%)^{* *}$ & $<0.001$ \\
\hline Volume of glomerulus $\left(\times 10^{6} \mu \mathrm{m}^{3}\right)$ & $4.97 \pm 0.71$ & $4.59 \pm 0.64$ & $5.13 \pm 0.81$ & $5.53 \pm 0.68^{*}$ & 0.031 \\
\hline Mean global sclerosis (\%) & $27.5 \pm 24.2$ & $29.1 \pm 25.9$ & $26.8 \pm 23.4$ & $25.1 \pm 21.7$ & 0.634 \\
\hline Mesangial expansion (0-3) & $2.51 \pm 1.22$ & $2.86 \pm 1.14$ & $2.33 \pm 1.25^{*}$ & $2.06 \pm 1.21^{* *}$ & 0.001 \\
\hline K-W nodule (\%) & $145(55.4 \%)$ & $81(66.4 \%)$ & $40(48.2 \%)^{*}$ & $24(40.7 \%)^{* *}$ & $<0.001$ \\
\hline Interstitial fibrosis (0-3) & $1.48 \pm 0.72$ & $1.57 \pm 0.68$ & $1.35 \pm 0.72$ & $1.48 \pm 0.87$ & 0.197 \\
\hline Tubular atrophy (0-3) & $1.07 \pm 0.21$ & $1.14 \pm 0.39$ & $1.04 \pm 0.37$ & $0.97 \pm 0.18^{*}$ & 0.012 \\
\hline
\end{tabular}

NOTE. Values were expressed as expressed as mean \pm SD and categorical data expressed as number (\%). Abbreviation: BMI, body mass index; K-W, Kimmelstiel-Wilson; $\mathrm{Ccr}$, creatinine clearance rate. To convert serum creatinine in $\mu \mathrm{mol} / \mathrm{L}$ to $\mathrm{mg} / \mathrm{dL}$, multiply by 0.0113 ; creatinine clearance rate in $\mathrm{mL} / \mathrm{s} / 1.73 \mathrm{~m}{ }^{2}$ to $\mathrm{mL} / \mathrm{min} / 1.73 \mathrm{~m}{ }^{2}$, multiply by 60.0 . The listed $P$ values are based on ANOVA or Chi-square test. ${ }^{*} P<0.05$ and ${ }^{* *} P<0.01$ versus lean group. ${ }^{\ddagger} P<0.01$ versus overweight group.

\section{Results}

\section{Obesity profile of DN patients in China}

All 264 patients were diagnosed with type 2 diabetes and renal biopsy-confirmed DN. Analysis of the baseline characteristics are shown in Figure 1 and Table 1. The mean age of patients with DN was $53.1 \pm 9.06$ years (range 31-80). Of the DN patients, 58.3\% were male. The average BMI was $25.5 \pm 3.39 \mathrm{~kg} / \mathrm{m}^{2}$ (range 18.936.6 ), and the frequency of BMI peaked at $24 \mathrm{~kg} / \mathrm{m}^{2}$ (Figure 1A). Among these patients, 22.3\% were obese, while $53.8 \%$ were obese/overweight. The average proteinuria was $3.09 \pm 2.32 \mathrm{~g} / 24 \mathrm{~h}$ (range 0.4-22.9), (Figure 1B), and $37.4 \%$ had nephrotic range proteinuria. Mean serum albumin was $34.5 \pm 7.4 \mathrm{~g} / \mathrm{L}$, and $53.5 \%$ had hypoalbuminemia. Renal Ccr greatly varied in patients and the frequency of $\mathrm{Ccr}$ peaked around $50 \mathrm{~mL} / \mathrm{s} /$ $1.73 \mathrm{~m}^{2}$ (Figure 1C). Renal insufficiency occurred in
$32.3 \%$ of subjects, $129(48.9 \%)$ patients remained classified as early stage (K/DOQI stage I), and no patients suffered from ESRD. The mean serum creatinine was $2.02 \pm 2.02 \mathrm{mg} / \mathrm{dL}$.

Patients were divided into three groups on the basis of BMI as follows: obese, $\geq 28.0 \mathrm{~kg} / \mathrm{m}^{2}(\mathrm{n}=59)$; overweight, $25-30 \mathrm{~kg} / \mathrm{m}^{2}(\mathrm{n}=83)$; and lean, $18-25 \mathrm{~kg} / \mathrm{m}^{2}(\mathrm{n}=122)$. The mean age and sex ratio among the three groups were similar, as were fasting glucose, HbA1c, mean blood pressure and previous therapy. At the time of diagnosis, the known duration of diabetes among patients ranged from a few months to 28 years. Compared with the obese patients, the lean patients suffered a longer duration of diabetes $(P<0.01)$, although the average history of proteinuria was similar among the three groups.

Among the lean patients, the percentage of renal insufficiency was $42.6 \%$, presenting Ccr less than $60 \mathrm{~mL} / \mathrm{s} /$ 

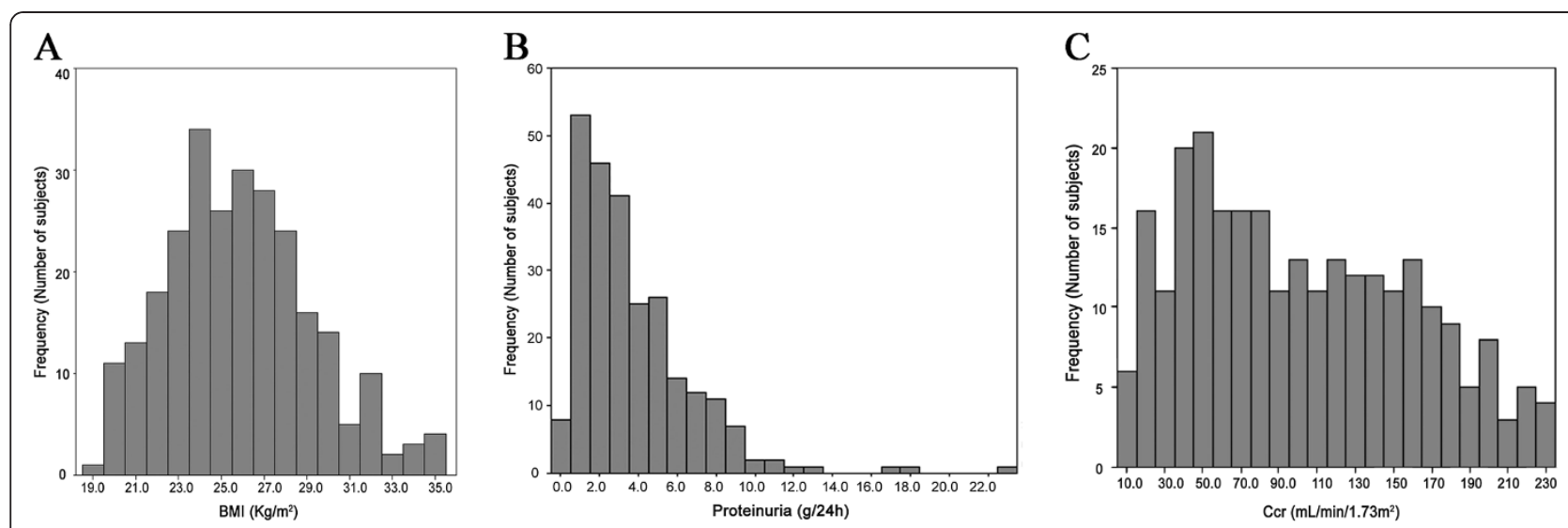

Figure 1 Histogram of weight mass index (A), proteinuria (B) and creatinine clearance rate (C) distribution among study subjects with diabetic nephropathy.

$1.73 \mathrm{~m}^{2}$, which was significantly higher than that in the obese or overweight patients $(P<0.001$, ANOVA). When compared with obese patients, the lean patients with $\mathrm{DN}$ showed higher levels of serum creatinine $(P=0.010)$, a higher percentage of hypoalbuminemia $(P=0.007)$ and anemia $(P<0.001)$, and greater mesangial expansion $(P<0.001)$, tubular atrophy $(P=0.012)$ and incidence of $\mathrm{K}-\mathrm{W}$ nodules $(P<0.001)$. Obese patients had larger glomeruli than those with a lean phenotype $(P=0.031)$.

\section{Obesity and improved renal survival in DN patients}

The information for follow-up was available for only 264 patients, and the median follow-up time was 39.0 months (range 0-87.0) (Figure 2). The median time of follow-up was 39.0, 36.0 and 36.0 months for the lean, overweight and obese groups, respectively. The levels of fasting glucose fluctuated but were similar among the three groups (Figure 2B). By the end of the study period, 74 cases (28.8\%) had developed ESRD.

Compared with obese patients, the lean patients had a greater risk for developing ESRD (Figure 2). After 36 months of follow-up, the mean increment of serum creatinine was $126 \%$ in the lean group, while this was $52.7 \%$ and $33.5 \%$ in the overweight and obese groups, respectively $(P<0.001$, ANOVA $)$. By the end of the study period, 48 (39.4\%) lean patients developed ESRD, and the relative risk of developing ESRD for the lean phenotype was 1.812 (95\% CI 1.004-3.268; $P=0.048$, Figure $2 C$ ). On the other hand, the mean BMI decreased among patients in the overweight or obese groups (Figure 2D), while no obvious change in BMI was detected in the lean group (data not shown). Regarding the obese patients, the reduction in BMI reached $1.30 \mathrm{~kg} / \mathrm{m}^{2}$ and $2.28 \mathrm{~kg} / \mathrm{m}^{2}$ after 36 and 63 months follow-up, respectively. The percentage of obesity in DN patients decreased from $22.3 \%$ at baseline to $19.3 \%$ at the end of the study period, while the rate of the lean phenotype increased from $46.2 \%$ to $58.3 \%$.

\section{Obesity and increased proteinuria at early stage DN}

Using the classification of chronic kidney disease, 129 subjects were classified as K/DOQI stage I and analyzed for an association between obesity and increased proteinuria at the early stage (Table 2). The demographic profile, including age and sex ratio, was similar between the total patient population studies and those at the early stage (data not shown). The patients at the early stage were further divided into three subgroups based on BMI: obese $(\mathrm{n}=32)$, overweight $(\mathrm{n}=51)$ and lean $(\mathrm{n}=46)$.

Among the patients at the early stage, the known duration of diabetes was similar among the three subgroups, although this factor differed in the total patient population studied and those with DN. The known duration of proteinuria was similar among the three groups at the early stage, as were the levels of fasting glucose, HbA1c and mean blood pressure. At the early stage, greater renal damage was observed in obese patients compared with lean patients, with greater glomerular hyperfiltration and a lower percentage of anemia $(P=0.027$ and $P=0.007$, respectively).

Among the patients at the early stage, the median time of follow-up was 30.0 months (Figure 3). The fasting glucose levels were almost stable during follow-up (Figure 3B). By the end of the study period, only 2 (1.55\%) cases developed ESRD, while 30 (24.8\%) patients had increased proteinuria. The mean levels of proteinuria increased in the obese patients, reaching $11.3 \%$ and 63.3\% after 36 and 63 months, respectively (Figure 3A). However, no obvious increase in proteinuria was detected in the lean and overweight subgroups. The relative risk for increased proteinuria was high in the obese patients, and $12(37.5 \%)$ exhibited a 50\% increase in 

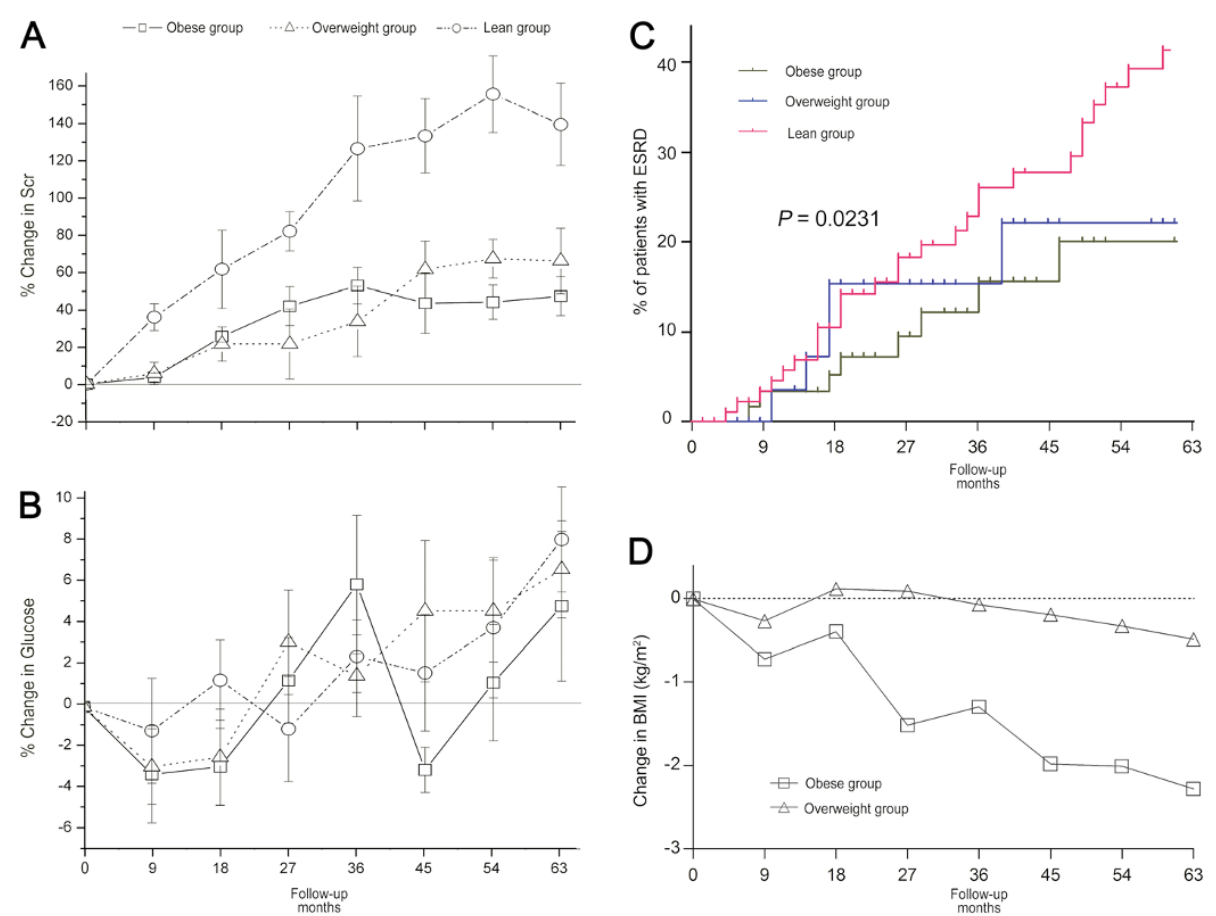

Figure 2 The follow-up of glucose control and renal lesions in patients with $D N(n=264)$ : A, mean change in Scr (\%); B, mean change in fasting glucose; $C$, cumulative incidence (\%) of end-stage renal disease: patients with obesity have improved renal survival compared those with lean BMI (Kaplan-Meier analysis, log-rank, $P=0.0231$ ); $\mathrm{D}$, mean change in BMI (\%).

proteinuria compared with the baseline levels. Using the lean group as a reference, obesity showed a significantly higher risk for increased proteinuria (Hazard ratio 2.872 [95\% CI 1.239-6.659], $P=0.014)$. Compared with the total population of obese patients studied, the decrease in mean BMI was relatively small in obese patients at the early stage $(P=0.018)$ and was only $1.12 \mathrm{~kg} / \mathrm{m}^{2}$ after 63 months of follow-up (Figure 3D). The overweight patients at the early stage did not show obvious changes in BMI.

\section{Discussion}

With rapid nutrition and lifestyle transitions over the past two decades, the global rate of obesity has been increasing at an alarming rate, including in China [19]. The frequency of diabetes has risen in tandem, as obesity is a component of risk factors for diabetes [20]. Today, China has the largest number of patients with obesity and diabetes mellitus in the world. Furthermore, increasing numbers of studies have investigated the relationship between obesity and DN in this ethnic group. This study shows for the first time that obesity and weight loss are common in Chinese patients with biopsyconfirmed DN.

Although all the patients were from a single unit, the profile of patients is representative of the characteristics of Chinese patients with renal disease, especially those in Eastern China. In our institute, there was an abrupt rise in the number of patients undergoing biopsy after 1985 , reaching 500 cases per year in 1991, with a further increase to over 1,000 cases per year after 1998 [9]. Recently, the number of renal biopsies performed each year has exceeded 2,000. The expanded pool of renal biopsies from kidney disease patients makes the epidemiologic investigation more reliable. However, the patients were not regularly interviewed in our institute because of concerns of economy and/or distance. The follow-up information, therefore, was relatively limited. Among all 757 patients with $\mathrm{DN}$, follow-up information was available for only 264 subjects. The baseline characteristics were similar between the 757 patients and 264 patients (data not shown).

In Chinese patients with biopsy-confirmed DN, 22.3\% presented with obesity and $53.7 \%$ with overweight/obesity. The prevalence of obesity was as high as $24.8 \%$ in patients with early stage DN. Compared with the prevalence in the general Chinese population [21], obesity was more common in the patients with DN. High prevalence of obesity has been confirmed in other ethnic groups [22]. The obese patients with DN in this study exhibited weight loss with diabetes during follow-up. On average, BMI decreased by $4.62 \%\left(2.28 \mathrm{~kg} / \mathrm{m}^{2}\right)$ after 63 -months in obese patients, while only moderate decreases were observed in patients at the early stage. This suggested 
Table 2 The profile of sub-cohort with a creatinine clearance $(\mathrm{Ccr})$ of more than $90 \mathrm{~mL} / \mathrm{min} / 1.73 \mathrm{~m}^{2}$

\begin{tabular}{|c|c|c|c|c|c|}
\hline & Total & Lean sub-group & Overweight sub-Group & Obese sub-Group & $P$ value \\
\hline$N$. of patients & 129 & 46 & 51 & 32 & \\
\hline Age (years) & $52.6 \pm 9.05$ & $53.1 \pm 9.36$ & $52.1 \pm 9.04$ & $52.5 \pm 8.82$ & 0.883 \\
\hline Male sex (\%) & $78(60.5 \%)$ & $28(60.9 \%)$ & $31(60.8 \%)$ & $19(59.3 \%)$ & 0.987 \\
\hline $\mathrm{BMI}\left(\mathrm{kg} / \mathrm{m}^{2}\right)$ & $25.9 \pm 3.05$ & $23.0 \pm 1.36$ & $26.4 \pm 0.84$ & $30.5 \pm 1.73$ & - \\
\hline $\mathrm{Ccr}\left(\mathrm{mL} / \mathrm{min} / 1.73 \mathrm{~m}^{2}\right)$ & $148 \pm 38$ & $138 \pm 31$ & $151 \pm 40$ & $159 \pm 42^{* *}$ & 0.027 \\
\hline$>150 \mathrm{~mL} / \mathrm{min} / 1.73 \mathrm{~m}^{2}(\%)$ & $93(72.1 \%)$ & $28(60.9 \%)$ & $37(72.5 \%)$ & $28(87.5 \%)^{*}$ & 0.036 \\
\hline Known duration of diabetes (months) & $83.6 \pm 68.8$ & $91.7 \pm 57.4$ & $80.8 \pm 63.8$ & $76.6 \pm 59.6$ & 0.746 \\
\hline Known duration of proteinuria (months) & $22.0 \pm 25.6$ & $20.0 \pm 25.3$ & $24.7 \pm 26.1$ & $20.5 \pm 26.7$ & 0.549 \\
\hline Fasting glucose (mg/dL) & $131 \pm 48$ & $134 \pm 52$ & $122 \pm 35$ & $143 \pm 60$ & 0.184 \\
\hline $\mathrm{HbA1c}(\%)$ & $6.81 \pm 1.46$ & $7.04 \pm 1.71$ & $6.48 \pm 1.21$ & $7.03 \pm 1.35$ & 0.133 \\
\hline Mean blood pressure $(\mathrm{mm} \mathrm{Hg})$ & $105 \pm 13.4$ & $106 \pm 15.3$ & $103 \pm 11.7$ & $105 \pm 12.9$ & 0.364 \\
\hline Proteinuria (g/24 h) & $2.36 \pm 2.33$ & $2.39 \pm 2.39$ & $2.29 \pm 2.32$ & $2.43 \pm 2.33$ & 0.901 \\
\hline$\geq 3.5 \mathrm{~g} / 24 \mathrm{~h}(\%)$ & $30(23.2 \%)$ & $9(19.6 \%)$ & $13(25.5 \%)$ & $8(25.0 \%)$ & 0.760 \\
\hline Serum albumin ( $g / d L)$ & $3.66 \pm 0.77$ & $3.47 \pm 0.74$ & $3.69 \pm 0.77$ & $3.78 \pm 0.81$ & 0.224 \\
\hline Serum creatinine $(\mathrm{mg} / \mathrm{dL})$ & $0.88 \pm 0.25$ & $0.89 \pm 0.21$ & $0.89 \pm 0.27$ & $0.86 \pm 0.24$ & 0.987 \\
\hline Hemoglobin (g/dL) & $12.7 \pm 2.2$ & $12.0 \pm 2.0$ & $12.8 \pm 2.2$ & $13.6 \pm 2.2$ & 0.016 \\
\hline Anemia (\%) & $33(25.6 \%)$ & $19(41.3 \%)$ & $10(19.6 \%)$ & $4(12.5 \%)$ & 0.007 \\
\hline High hemoglobin (\%) & $17(13.2 \%)$ & $5(10.9 \%)$ & $6(11.8 \%)$ & $6(18.8 \%)$ & 0.338 \\
\hline Volume of glomerulus $\left(\times 10^{6} \mu \mathrm{m}^{3}\right)$ & $5.45 \pm 0.0 .55$ & $4.79 \pm 0.31$ & $5.44 \pm 0.57^{*}$ & $6.40 \pm 0.71^{* *}$ & 0.014 \\
\hline Meanglobal sclerosis (\%) & $16.3 \pm 16.4$ & $13.3 \pm 13.6$ & $15.7 \pm 17.0$ & $21.7 \pm 19.6$ & 0.178 \\
\hline Mesangial expansion (0-3) & $1.70 \pm 0.80$ & $1.96 \pm 0.84$ & $1.54 \pm 0.78$ & $1.62 \pm 0.59$ & 0.698 \\
\hline K-W nodule (\%) & $55(42.6 \%)$ & $27(58.7 \%)$ & $18(35.3 \%)^{*}$ & $10(31.3 \%)^{*}$ & 0.012 \\
\hline Interstitial fibrosis (0-3) & $1.20 \pm 0.66$ & $1.62 \pm 0.64$ & $1.50 \pm 0.59$ & $1.23 \pm 0.31$ & 0.430 \\
\hline Tubular atrophy (0-3) & $0.98 \pm 0.30$ & $1.05 \pm 0.32$ & $0.95 \pm 0.30$ & $0.94 \pm 0.24$ & 0.267 \\
\hline
\end{tabular}

NOTE. Values were expressed as mean \pm SD or number (percentage); Abbreviation: BMI, body mass index; K-W, Kimmelstiel-Wilson; Ccr, creatinine clearance rate. To convert serum creatinine in $\mathrm{mol} / \mathrm{L}$ to $\mathrm{mg} / \mathrm{dL}$, multiply by 0.0113 ; creatinine clearance rate in $\mathrm{mL} / \mathrm{s} / 1.73 \mathrm{~m}{ }^{2}$ to $\mathrm{mL} / \mathrm{min} / 1.73 \mathrm{~m}{ }^{2}, \mathrm{multiply}$ by 60.0 . The listed $P$ values are based on ANOVA or Chi-square test. ${ }^{*} P<0.05$ and ${ }^{* *} P<0.01$ versus lean group.

that weight loss was a predictive marker of poor or declining health for diabetic patients, which was consistent with the findings of other studies [23]. Accordingly, the patients with lean phenotype had a longer duration of diabetes than those with obesity. Compared with obese patients, lean patients exhibited more severe renal injury, including higher percentage of renal insufficiency and a higher score for tubular lesions. Taking the lean group as the reference, obesity and overweight significantly decreased the risk for ESRD. This confirmed that a proportion of lean patients suffer from both DN development and weight loss before renal biopsy, and indicates that weight loss is the major characteristics of the natural course of DN and shows a predictive effect on DN in China. Thus, the lean phenotype indicates disease progression. However, a protective role for obesity in renal disease progression was not identified in this retrospective study.

On the other hand, weight control was generally thought to decrease the risk for renal injury associated with obesity and diabetes. Our previous study also proved that weight loss decreased urinary protein by $51 \%$ after 24 months in subjects with obesity-related glomerulopathy (ORG) [24]. Saiki et al. reported the protective effect of weight loss using a formula diet on renal function in obese patients with $\mathrm{DN}$ [25]. These results contrast with our findings. In fact, more studies have suggested the "obesity paradox" in diabetes and renal diseases [26]. In the present study, a lean phenotype and weight loss were associated with a "deterioration phenomenon" and progression of renal injury in DN patients in China. On the other hand, obesity contributed to increased proteinuria at the early stage of DN. Among patients at CKD stage I, obese patients showed greater hyperfiltration than lean patients. Obesity further increased the risk for increased proteinuria by 2.872 compared with the lean patients. Similar results were found in the subjects with normal renal function or at the early stage of disease. Thus, the present study using one population of DN patients showed a varied effect of obesity and weight loss on the progression of DN. 

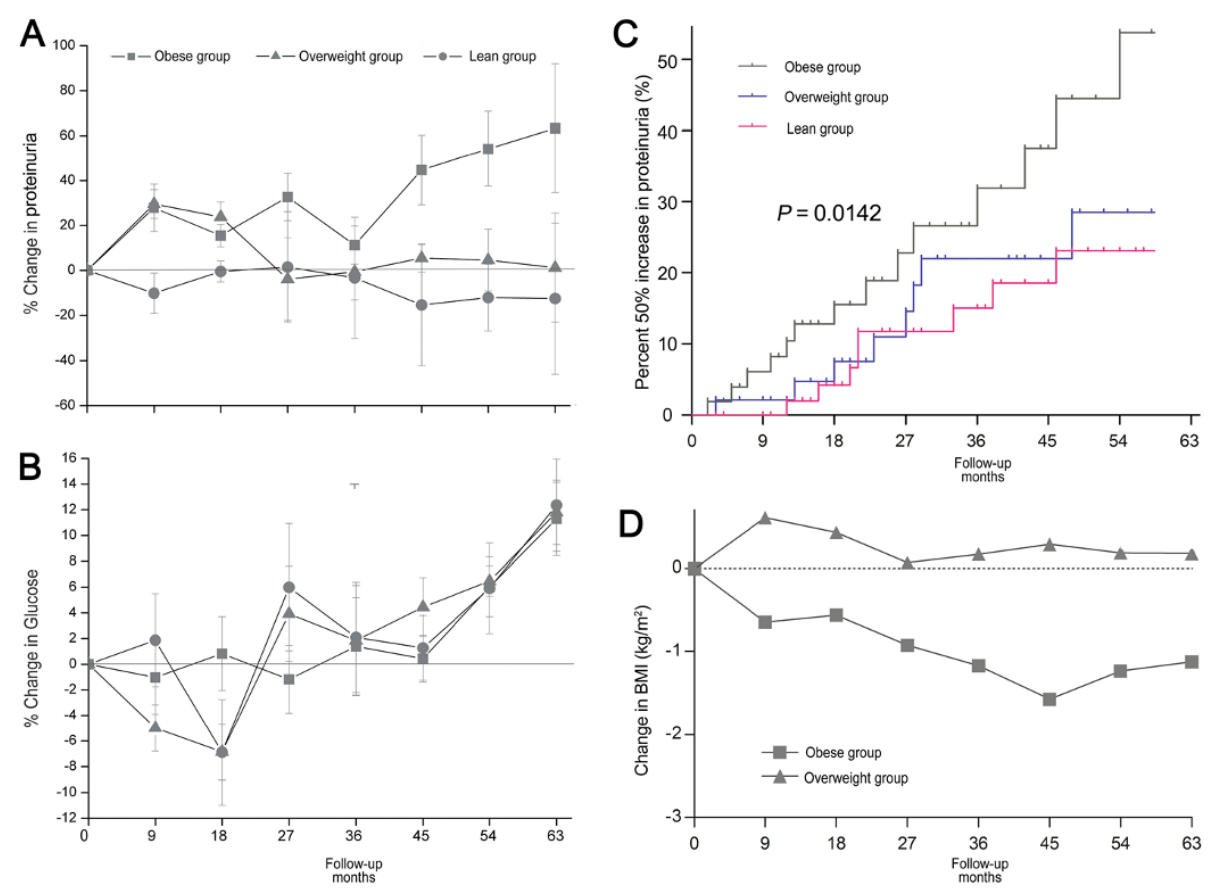

Figure 3 The follow-up of glucose control and renal lesions in subjects at K/DOQI stage I $(\mathbf{n}=129)$. A, mean change in proteinuria (\%); B, mean change in fasting glucose; C, cumulative incidence (\%) of proteinuria progression: patients with obesity have higher risk of progress of proteinuria compared those with lean BMI (Kaplan-Meier analysis, log-rank, $P=0.0142$ ); $\mathbf{D}$, mean change in BMI (\%).

Much evidence has shown that the different effect of weight loss resulted from two different mechanisms of weight loss: intentional and unintentional. In brief, diet control and extra exercise leads to intentional weight loss, mainly from the fat mass, thus improving insulin resistance, inflammation, and subsequent renal injury. However, with the progression of diabetes, high sugar levels lead to dehydration, muscle breakdown and an unintentional weight loss, which predicted clinical deterioration. Zoccali [27] suggested that maintenance of the current weight was the best way to protect against DN. Unfortunately, BMI was the only marker used to evaluate obesity in the present study, and it was difficult to classify the source of weight loss. As a retrospective study, the different effect of weight loss on DN was suggested by the "obesity paradox" with no direct evidence in support of this phenomenon. Further studies, especially prospective cohort studies, are required to compare the effect of intentional and unintentional weight loss on DN, which might help to explain the "obesity paradox" and "weight loss paradox" in China.

It should be pointed out that there are several additional limitations associated with this retrospective study. Serum creatinine levels were used in the present study to define ESRD, although this is not a sufficient measure of the loss of renal function, as it can be influenced by muscle mass, nutritional status and gender. In our institute, patients are usually diagnosed with ESRD, when serum creatinine levels exceed $6 \mathrm{mg} / \mathrm{dL}$ for more than 1 month, and other causes of renal disease are excluded. The value of " $6 \mathrm{mg} /$ $\mathrm{dL}$ " was adopted as a "cut-off" based on the value commonly used in Chinese medical schools [28]. Although insufficient, serum creatinine is a conveniently tested measure in clinical practice, and was therefore used as a marker of the loss of renal function.

Because of the limitations of this study in terms of population number and follow-up information, the development of ESRD does not reflect the progression of renal injury in all patients with $\mathrm{DN}$. According to the different features of $\mathrm{DN}$ at different stages, two endpoints were used to assess the progression of $\mathrm{DN}$ in the present study. The development of ESRD was used as the first endpoint, and increased proteinuria was used as the second endpoint only for those patients at the early stage. It is well known that early stage patients exhibit micro-albuminuria or moderate proteinuria, with normal levels of serum creatinine [10]. The levels of albuminuria/proteinuria were shown to be associated with the severity and out-come of renal injury. At a later stage, most patients had gross proteinuria and increased serum creatinine. High levels of serum creatinine are linked with ESRD, while the levels of proteinuria fluctuate with diet and other factors. Using diverse end-points, our study shows that obesity is significantly associated with increased proteinuria in early stage patients, and the lean phenotype is linked with ESRD in all DN patients. 
It should also be noted that a direct effect of obesity has been shown on renal function contributing to proteinuria and renal lesions [29]. Some obese patients probably suffered from ORG prior to the diagnosis of type 2 diabetes or DN. It is difficult to distinguish between these two diseases based on natural history, or from histological features. ORG is characterized by glomerulomegaly and focal segmental glomerulosclerosis, which can also be observed in DN. Patients with DN, especially obese subjects, might present with ORG simultaneously. Previous ORG and/ simultaneous ORG partly added to the difference in progression of CKD between lean and overweight/obese patients.

\section{Conclusions}

Overall, our study demonstrates that obesity is very common among DN patients undergoing renal biopsy in China, which is associated with the most rapid increases in both obesity and diabetes globally. Obese patients showed a natural course of DN - heavy proteinuria initially, followed by weight loss. Obesity seemed to be associated with increased proteinuria at the early stage, while it was associated with a benefit in terms of improved renal survival at the later stages. Weight loss was accompanied by the progression of diabetic nephropathy with a prolonged diabetic duration. Lean patients exhibited a greater acceleration of disease toward ESRD than obese patients. The present study further elucidates the mechanism of progression of $\mathrm{DN}$ in China in relation to obesity and warns of the concurrence of weight loss and the development of diabetes. Regular evaluation of weight is important for the patients with $\mathrm{DN}$, as well monitoring of glucose levels, proteinuria and serum creatinine.

\section{Additional file}

Additional file 1: Table S1. Epidemiological characterisstics of subjects with diabetic nephropathy. NOTE. Values were expressed as expressed as mean \pm SD and categorical data expressed as number (\%). Abbreviation: $\mathrm{BMl}$, body mass index. To convert serum creatinine in $\mu \mathrm{mol} / \mathrm{L}$ to $\mathrm{mg} / \mathrm{dL}$, multiply by 0.0113 ; creatinine clearance rate in $\mathrm{mL} / \mathrm{s} / 1.73 \mathrm{~m}^{2}$ to $\mathrm{mL} / \mathrm{min} /$ $1.73 \mathrm{~m}^{2}$, multiply by 60.0. The listed $P$ values are based on student's test or Chi-square test. ${ }^{*} P<0.05$ and $^{* *} P<0.01$ versus lean group. ${ }^{*} P<0.01$ versus overweight group.

\section{Competing interests}

The authors declare that they have no competing interest.

\section{Authors' contribution}

CHM and LZH designed the study. SWW, GYC and ZYD collected samples and clinical information. XHL performed the laboratory assays. CHM performed the statistical analyses and wrote the manuscript. The final version of the manuscript was approved by all authors.

\section{Acknowledgment}

This work was partly supported by the National Natural Science Foundation of China (grant number: 81070579) and the Six Talent Peaks Project of Jiangsu Province (grant number: 2012-WSN-071). The funders had no role in study design, data collection and analysis, decision to publish, or preparation of the manuscript. No additional external funding received for this study.

Received: 16 August 2012 Accepted: 13 March 2013

Published: 25 March 2013

\section{References}

1. $\mathrm{Ji} \mathrm{CY}$, Cheng TO: Epidemic increase in overweight and obesity in Chinese children from 1985 to 2005. Int J Cardiol 2009, 132(1):1-10.

2. Hsu CY, McCulloch CE, Iribarren C, Darbinian J, Go AS: Body mass index and risk for end-stage renal disease. Ann Intern Med 2006, 144(1):21-28.

3. Hsieh MC, Hsieh YT, Cho TJ, Chen JF, Lin SD, Chen HC, Tu ST: Remission of diabetic nephropathy in type 2 diabetic Asian population: role of tight glucose and blood pressure control. Eur J Clin Invest 2011, 41(8):870-878.

4. Luk A, Chan JC: Diabetic nephropathy-what are the unmet needs? Diabetes Res Clin Pract 2008, 82(Suppl 1):S15-S20.

5. Speakman JR, Westerterp KR: Reverse epidemiology, obesity and mortality in chronic kidney disease: modelling mortality expectations using energetics. Blood Purif 2010, 29(2):150-157.

6. Valocikova I, Valocik G, Kristofova B, Druzbacka L: Obesity paradox and chronic kidney disease. Bratis/ Lek Listy 2011, 112(7):402-406.

7. Pei YP, Greenwood CM, Chery AL, Wu GG: Racial differences in survival of patients on dialysis. Kidney Int 2000, 58(3):1293-1299.

8. Zhou BF: Predictive values of body mass index and waist circumference for risk factors of certain related diseases in Chinese adults-study on optimal cut-off points of body mass index and waist circumference in Chinese adults. Biomed Environ Sci 2002, 15(1):83-96.

9. Li LS, LiU ZH: Epidemiologic data of renal diseases from a single unit in China: analysis based on 13,519 renal biopsies. Kidney Int 2004, 66(3):920-923.

10. Alberti KG, Zimmet PZ: Definition, diagnosis and classification of diabetes mellitus and its complications. Part 1: diagnosis and classification of diabetes mellitus provisional report of a WHO consultation. Diabet Med 1998, 15(7):539-553.

11. Jennette JLO JC, Schwartz MM, Silva FG: Heptinstall's Pathology of the Kidney. 5th edition. Lippincott Willians \& Wirkins; 1998.

12. Huang F, Yang Q, Chen L, Tang S, Liu W, Yu X: Renal pathological change in patients with type 2 diabetes is not always diabetic nephropathy: a report of 52 cases. Clin Nephrol 2007, 67(5):293-297.

13. Tone A, Shikata K, Matsuda M, Usui H, Okada S, Ogawa D, Wada J, Makino $\mathrm{H}$ : Clinical features of non-diabetic renal diseases in patients with type 2 diabetes. Diabetes Res Clin Pract 2005, 69(3):237-242.

14. Lemann J, Bidani AK, Bain RP, Lewis EJ, Rohde RD: Use of the serum creatinine to estimate glomerular filtration rate in health and early diabetic nephropathy. Collaborative study group of angiotensin converting enzyme inhibition in diabetic nephropathy. Am J Kidney Dis 1990, 16(3):236-243.

15. Chen HM, Chen Y, Zhang YD, Zhang PP, Chen HP, Wang QW, Li LS, Liu ZH: Evaluation of metabolic risk marker in obesity-related glomerulopathy. J Ren Nutr 2011, 21(4):309-315.

16. Lane PH, Steffes MW, Mauer SM: Estimation of glomerular volume: a comparison of four methods. Kidney Int 1992, 41(4):1085-1089.

17. Chen HM, Liu ZH, Zeng CH, Li SJ, Wang QW, Li LS: Podocyte lesions in patients with obesity-related glomerulopathy. Am J Kidney Dis 2006, 48(5):772-779.

18. Gellman DD, Pirani CL, Soothill JF, Muehrcke RC, Kark RM: Diabetic nephropathy: a clinical and pathologic study based on renal biopsies. Medicine (Baltimore) 1959, 38:321-367.

19. Weight loss helps type 2 diabetes patients. Dis Manag Advis 2008, 14(10):11-12.

20. Ramachandran A, Ma RC, Snehalatha C: Diabetes in Asia. Lancet 2010, 375(9712):408-418.

21. Wu Y: Overweight and obesity in China. BMJ 2006, 333(7564):362-363.

22. Maric C, Hall JE: Obesity, metabolic syndrome and diabetic nephropathy. Contrib Nephrol 2011, 170:28-35.

23. Meltzer AA, Everhart JE: Unintentional weight loss in the United States. Am J Epidemiol 1995, 142(10):1039-1046.

24. Shen WW, Chen HM, Chen H, Xu F, Li LS, Liu ZH: Obesity-related glomerulopathy: body mass index and proteinuria. Clin J Am Soc Nephrol 2010, 5(8):1401-1409. 
25. Saiki A, Nagayama D, Ohhira M, Endoh K, Ohtsuka M, Koide N, Oyama T, Miyashita Y, Shirai K: Effect of weight loss using formula diet on renal function in obese patients with diabetic nephropathy. Int J Obes (Lond) 2005, 29(9):1115-1120.

26. Andreotti F, Rio T, Lavorgna A: Body fat and cardiovascular risk: understanding the obesity paradox. Eur Heart J 2009, 30(7):752-754.

27. Zoccali C: The obesity epidemics in ESRD: from wasting to waist? Nephrol Dial Transplant 2009, 24(2):376-380.

28. Wang Y: Medicine. Houst, co: People's Medical Publish; 2010

29. Chen HM, Li SJ, Chen HP, Wang QW, Li LS, Liu ZH: Obesity-related glomerulopathy in China: a case series of 90 patients. Am J Kidney Dis 2008, 52(1):58-65.

doi:10.1186/1471-2369-14-69

Cite this article as: Chen et al:: The relationship between obesity and diabetic nephropathy in China. BMC Nephrology 2013 14:69.

\section{Submit your next manuscript to BioMed Central and take full advantage of:}

- Convenient online submission

- Thorough peer review

- No space constraints or color figure charges

- Immediate publication on acceptance

- Inclusion in PubMed, CAS, Scopus and Google Scholar

- Research which is freely available for redistribution 